\title{
Pulmonary valve replacement for congenital heart disease: What valve substitute should we be using?
}

\author{
Max B. Mitchell, MD
}

\footnotetext{
From the University of Colorado at Denver Health Sciences Center and The Children's Hospital Colorado Heart Institute, Aurora, Colo.

Disclosures: Author has nothing to disclose with regard to commercial support.

Received for publication July 6, 2016; accepted for publication July 12, 2016; available ahead of print Sept 13, 2016.

Address for reprints: Max B. Mitchell, MD, University of Colorado at Denver Health Sciences Center and The Children's Hospital Colorado Heart Institute, Aurora, CO 80045 (E-mail: max.mitchell@ childrenscolorado. org).

J Thorac Cardiovasc Surg 2016;152:1230-2

$0022-5223 / \$ 36.00$

Copyright (c) 2016 by The American Association for Thoracic Surgery

http://dx.doi.org/10.1016/j.jtcvs.2016.07.031
}

Invited editorial for Nomoto et al. Outcomes of Stented Bovine Pericardial Tissue Valves for Pulmonary Valve Replacement in Congenital Heart Disease.

Progress in congenital heart surgery often results in the creation of patient populations in need of future surgical and catheter-based interventions. The treatment of tetralogy of Fallot and other forms of congenital heart disease involving the right ventricular outflow tract are good examples of this phenomenon. Pulmonary valve replacement (PVR) has become one of the most commonly performed procedures in congenital heart surgery. Despite the prevalence of this procedure, considerable uncertainty remains as to the indications and timing for surgery and which valve replacement option is most optimal. The group at Boston Children's Hospital has had a significant impact in defining the appropriate indications and timing for PVR for patients with congenital heart disease. The Boston group has also made contributions that have influenced the choice of valve substitutes for PVR that are in common use. Current tissue valve options for surgical PVR include allograft tissue valves (pulmonary or aortic), a bovine jugular vein valved conduit (ie, Contegra), stented porcine aortic valves, stentless porcine aortic valves, stented bovine pericardial tissue valves, and now stentless bovine pericardial tissue valves. Excluding allograft valves and the Contegra, most tissue valve types are available from several manufacturers, each having unique design and processing methods. On the plus side, we are blessed with a large variety of choices for PVR. Unfortunately, there are insufficient outcomes data to determine the best valve substitute for PVR. This conclusion is supported by the wide variety of valve choices currently used for PVR at institutions across the United States and the world. Patient factors such as age, size, diagnosis, previous surgical history, and right ventricular outflow tract anatomy, among other factors, influence this choice, and there is no single best solution for all patients. PVR is almost entirely indicated for congenital heart disease. Given the relatively

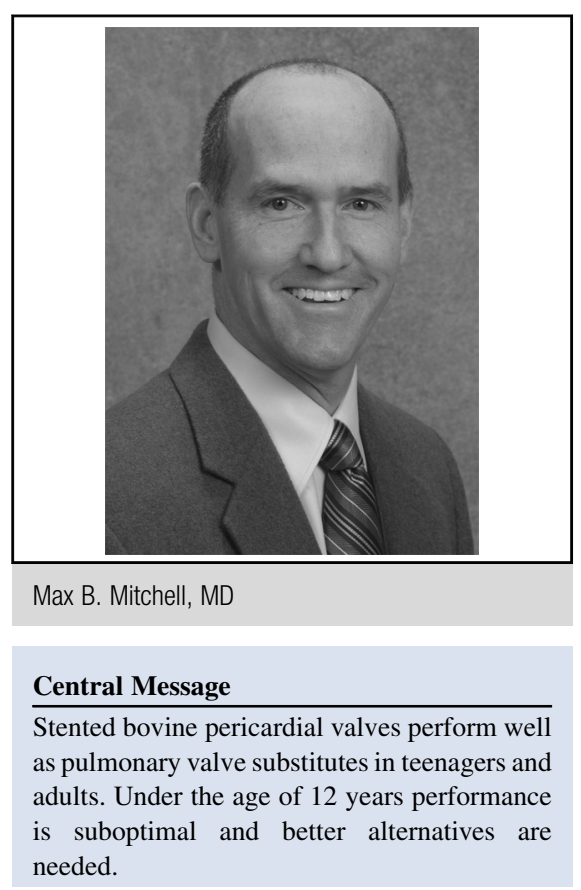

See Article page 1333. small number of patients requiring PVR, compared with aortic valve replacement for acquired disease, the available valve options are largely borrowed from aortic valve options not requiring anticoagulation. Stented bovine pericardial aortic valves offer a larger effective valve orifice compared with similar-sized stented porcine aortic valves with at least equivalent valve durability. These properties led to a significant increase in the use of stented bovine pericardial valves for acquired aortic valve disease in recent decades. Concurrently, suboptimal outcomes for allograft PVR were recognized. As a result, stented bovine pericardial valves were widely adopted for PVR in many centers beginning in the mid-1990s.

In this issue of the Journal of Thoracic and Cardiovascular Surgery, Nomoto and colleagues ${ }^{1}$ present the largest single-center series of PVRs using stented bovine pericardial valves published to date. The authors' stated purpose was to determine the outcomes of PVR in these patients, and the indications for PVR and timing of PVR are not addressed. Based on this study, one can assume that during the course of patient accrual (1996-2014) bovine pericardial valves became the dominant choice for PVR at 
Boston Children's Hospital, excluding neonates and young infants. I congratulate the authors for undertaking a comprehensive analysis on their large population of patients. This study is a significant contribution to the congenital cardiac surgical community, and the authors have added significantly to our knowledge.

Focusing on specifics, the authors report outcomes of 611 patients who had stented bovine pericardial valves for PVR over an 18-year period. Four stented bovine pericardial valves were used over roughly 3 near-equal time intervals. For purposes of analysis, the authors combined 2 similar valves from the same manufacturer into 1 group resulting in 3 valve types to compare. The CE PERIMOUNT (Edwards Life Sciences Corporation, Irvine, Calif; $[\mathrm{n}=72$, $11 \%]$ ) was used primarily during the first third of the study. The CE Magna/Magna Ease (Edwards Life Sciences Corporation, Irvine, Calif; [ $n=223,35 \%$ ]) was used primarily in the middle third, and to a lesser extent during the last third. The Sorin Mitroflow LXE (Sorin Group Canada, Inc. Burnaby, British Columbia, Canada; $[\mathrm{n}=315,50 \%]$ ) was the primary valve used during the most recent third of the study. Median follow-up for the series was 3.0 years. Importantly, the primary endpoint in the study was subsequent valve replacement (surgical PVR or catheter-deployed Melody PVR). The analysis of potential patient and valve factors is exhaustive. The authors assessed virtually every possible measure of body size for those patients under the age of 20 years including weight for age $\mathrm{Z}$ scores and body mass index (BMI) for age $\mathrm{Z}$ scores in an effort to determine the impact of body size on the primary outcome. Some of the study findings were expected, but other findings were unexpected and novel. The key findings were as follows. (1) Re-intervention rates varied by age with a significantly shorter time to re-intervention in younger patients compared with older patients regardless of valve type. (2) Regardless of age, re-intervention rates varied by valve type with a substantially shorter time to re-intervention in patients receiving the Sorin Mitroflow LXE valve. The 3-year re-intervention rate for the Mitroflow was $5 \%$. (3) By univariate analysis, all measures of younger age were risk factors for re-intervention. (4) A novel finding was that children with a low BMI for age $\mathrm{Z}$ score had an increased the risk for re-intervention with all other factors being constant. (5) Measures of larger valve size were protective against re-intervention. (6) A smaller body surface area-adjusted valve orifice area was protective against re-intervention.

The strong associations of younger age and smaller size with shorter valve durability mirror what is known with virtually all valve replacements done in the congenital cardiac population. While these associations are expected, 1 important facet of this study is illustrated in Figure 1. Freedom from re-intervention in adults and older pediatric patients was quite good: out to 8 years. However, there appears to be an age-related inflection point for 5-year outcomes with patients under the age of 12 years having a much lower incidence of freedom from re-intervention compared with older patients. The statistical methods used controlled for patient and valve size. An unaddressed but logical question inferred from this finding is whether or not stented bovine pericardial valves are a reasonable choice for patients under the age of 12 years who require PVR. At this juncture, there are no direct comparisons between bovine pericardial valves and other options (including available porcine bioprostheses and Contegra) in this age group. The relatively poor performance of bovine pericardial valves in patients less than 12 years indicates that such comparisons are sorely needed. This inference would also be applicable to the group of patients with small BMI relative to age.

A second significant and unexpected finding was the relatively poor performance of the Sorin Mitroflow LXE compared with the CE Magna/Magna Ease and CE PERIMOUNT valves. The Mitroflow LXE was used in smaller and younger patients. However, even with these factors controlled, the poor performance of the Mitroflow LXE persisted. One potential reason for this finding is that the Mitroflow LXE was manufactured without anticalcification treatment. This is a reasonable conclusion, but the CE PERIMOUNT valve also lacks anticalcification treatment and yet the CE PERIMOUNT performed as well if not better than the newer treated $\mathrm{CE}$ valves. Referring to Figure E1, 2 relevant observations stand out that may have influenced the findings. First, there was a large increase in PVRs/year performed after 2007, and more than half the study patients were accrued in the most recent third of the study. Program growth and a lower threshold for performing PVR in recent years are the 2 most likely explanations for this trend. The lower age at PVR in the latter third of the study suggests that the indications for PVR changed over time with a more aggressive approach adopted in the latter years of the study. Changing indications for intervention over time is expected particularly when new technology becomes available. The US Melody IDE trial began in 2007, and the availability of this valve almost certainly lowered the threshold for re-intervention after PVR due to the perceived lower impact of this treatment compared with repeat surgical PVR. This potentially confounds the findings because re-intervention for repeat surgical or non-surgical PVR was the primary endpoint used to measure outcome. The second notable observation in Figure E1 is that the distribution of valves used over time changed; most of valves used between 2008 and 2014 were the Mitroflow LXE. Coincidentally, the Mitroflow LXE was approved for the US market in 2007. In contrast, the CE Magna/Magna Ease valves were more evenly distributed between 2000 and 2014. Consequently, it is possible that a change in re-intervention threshold 
introduced by the availability of the Melody valve may have had a disproportionate and adverse impact on the performance of the Mitroflow LXE valve. In any case, the poor performance of the Mitroflow LXE valve is now only relevant to patients in whom this valve was implanted. Moving to the future, the LXE valve was replaced on the US market by the Mitroflow with phospholipid reduction treatment (PRT) valve in early 2014. The Mitroflow PRT valve is manufactured with a newer form of anticalcification treatment. Thus, the readership should be aware that the poor performance of the LXE valve cannot be generalized to the newer PRT valve.

All retrospective studies require compromises and possess significant limitations. As noted above, this study is no exception. The authors appropriately recognize the limitations of their study. Despite these limitations, their study adds significantly to the literature, and should affect our thinking in approaching patients requiring PVR. This paper strengthens my personal bias that bovine pericardial valves are a good option for teenagers and adults requiring PVR, but that other options including the Contegra and porcine aortic valves should be considered for patients under the age of 12 years. When this manuscript was presented at the 2015 annual meeting of The American
Association for Thoracic Surgery, an additional small group of $22(4 \%)$ early patients receiving porcine bioprosthetic PVR were included. Interestingly, the porcine group had the lowest median age of all groups, and yet their outcomes were among the most favorable. Unfortunately, the porcine bioprosthetic valve group was too small to permit valid comparison with the stented bovine pericardial valves groups and was a necessary casualty of the review process. Nevertheless, the information presented in oral form and absent from this manuscript suggests that efforts to compare porcine bioprosthetic options with stented bovine pericardial valves, particularly for patients under the age of 12 years, are greatly needed. The same is true of the need to compare Contegra outcomes with stented bioprostheses. Based on this study and current knowledge, stented bovine pericardial valves are a reasonably good option for teenage and adult patients with congenital heart disease. However, outcomes in children under the age of 12 years are significantly worse and other options should be considered.

\section{Reference}

1. Nomoto R, Sleeper LA, Borisuk MJ, Bergerson L, Pigula FA, Emani S, et al. Outcome and performance of bioprosthetic pulmonary valve replacement in patients with congenital heart disease. J Thorac Cardiovasc Surg. 2016;152:1333-42. 